\title{
Post-choice revaluation of hedonic preferences: Insights from functional imaging
}

\author{
Vinod Venkatraman ${ }^{1,2 * t}$, Maureen Ritchey ${ }^{1,2+}$ and Crystal Reeck ${ }^{1,2+}$ \\ Center for Cognitive Neuroscience, Duke University, Durham, NC, USA \\ Department of Psychology and Neuroscience, Duke University, Durham, NC, USA \\ *Correspondence: vinod.venkatraman@duke.edu \\ ${ }^{+}$All authors contributed equally to this work
}

\section{A commentary on}

How choice reveals and shapes expected hedonic outcome.

by Sharot, T., De Martino, B., and Dolan, R. J. (2009). J. Neurosci. 29, 3760-3765.

One of the principal goals of cognitive neuroscience is to illuminate aspects of psychological processes that are inaccessible to behavioral approaches. In many cases, neural data can promote breakthroughs in scientific understanding of psychological phenomena by facilitating adjudication between competing theories, implicating previously unknown mediating processes, or providing new mechanistic accounts of behavior. One such phenomenon that has been the focus of much investigation in social psychology is the effect of choice on perceived value. After being forced to choose between two equally valued options, such as two highly similar appliances, most people will subsequently rate their selected option more positively and the rejected option more negatively than they would have originally (Brehm, 1956). While this effect has been well-documented in the behavioral literature, little is known about the mechanisms that drive this alteration in perceived value. For example, these shifts in preference could indicate a need to reduce cognitive dissonance, or represent memory and context-dependent changes in stimulus representation during the decision period. Understanding the neural correlates associated with post-choice revaluations can help provide valuable insights in adjudicating between these mechanisms.

In a recent edition of the Journal of Neuroscience, Sharot et al. (2009) sought to delineate the neural mechanisms underlying post-choice revaluation of expected hedonic value using functional neuroimaging. Participants initially rated on a 6-point scale how happy they would be travelling to 80 different vacation destinations. Subsequently, participants chose between these destinations presented in pairs. Critically, these pairs were personalized for each participant such that one-third involved two destinations they had initially rated very differently and two-thirds combined destinations they had rated equally. Consistent with existing social psychology literature, participants rated the selected destinations as more pleasant and the rejected destinations as less pleasant after being forced to choose between pairs of destinations that had initially been rated equally (Sharot et al. 2009, Figure 1).
For the pairs that had initially been rated disparately, there was no change in the ratings post-choice.

Despite the lack of differences in ratings pre-choice, neuroimaging revealed a region in the caudate nucleus that tracked preference for the selected option even before revaluation was induced by the decision (Sharot et al. 2009, Figure 3b). The authors also demonstrated a further increase in activation for the selected option over rejected option post-choice within the right caudate (Sharot et al. 2009, Figure 3c). Finally, the post-choice increase in activation difference between the selected and

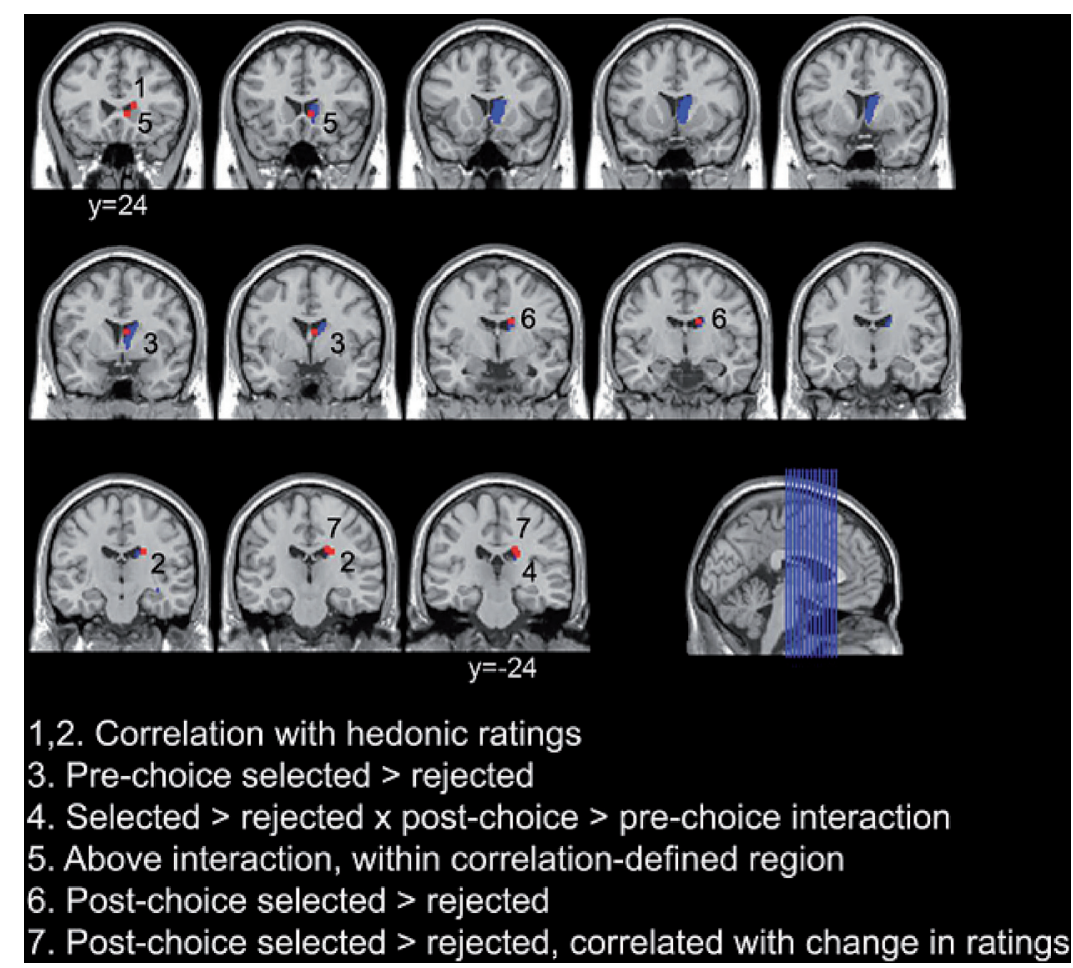

FIGURE 1 | Illustration of the anatomical variability in activation of the caudate nucleus across the various contrasts in Sharot et al. (2009) study. Voxel-sized boxes $(2 \times 2 \times 3 \mathrm{~mm})$ centered on the peak co-ordinates for each of the contrasts are overlaid in red on the SPM5T1 template brain. The right caudate nucleus, defined according to the Talairach Daemon atlas using the WFU PickAtlas tool, is additionally overlaid in blue. Slices are displayed from $y=-24$ to $y=24$ in increments of $4 \mathrm{~mm}$. 
rejected options predicted the magnitude of behavioral changes in valuation across individuals (Sharot et al. 2009, Figure 3d). Since a similar region in the caudate nucleus tracked preference ratings for the different destinations in an independent parametric analysis (after controlling for arousal, vividness and familiarity), the authors associate activation in the caudate nucleus with expected hedonic value.

This well-controlled study makes a significant contribution to the existing behavioral literature. First, it uses a clever adaptation of traditional behavioral paradigms used to study post-choice revaluation. Previous experiments often employed a limited number of decisions, while this study was able to assess numerous decisions, making it better suited for neuroimaging methods. Additionally, Sharot and colleagues demonstrate that activation in the caudate nucleus tracks decision preferences before participants even know they will choose between two items. These findings highlight the importance of using neuroimaging methods to provide insights into cognitive processes that may be inaccessible to typical behavioral measures.

While the results of this study are illuminating, it is a little surprising that hedonic value in this task is associated with activation in the caudate nucleus. In most studies, the ventromedial prefrontal cortex and ventral striatum have often been associated with computing values and tracking hedonic preferences (Hare et al., 2008; Seymour et al., 2007) while the caudate nucleus has often been associated with reinforcement of an action rather than the rewards themselves (Balleine et al., 2007; O'Doherty et al., 2004). Additionally, interpreting the functional role of the caudate nucleus in this study is further complicated by the vast anatomical variability across the different contrasts performed (see Figure 1). Since other studies provide evidence for functional specialization within the various regions of the caudate (Balleine et al., 2007; Yin and Knowlton, 2006), it is necessary to resolve this anatomical variability to fully appreciate the mechanisms underlying valuation of stimuli pre- and post-choice.

While this experiment provides insight into the post-choice revaluation of options, it does little to clarify the various mechanisms by which these alterations in perceived value might occur. One such account appeals to cognitive dissonance theory. Choosing between two equally attractive alternatives typically causes cognitive discomfort since the beneficial aspects of the rejected option and the limitations of the selected option conflict with the decision. In order to resolve this tension, both stimuli are revalued. An alternative explanation suggests that these revaluations are driven by contextual or memory-driven processes that occur during the choice period rather than the reduction of cognitive dissonance. Participants may retrieve additional new information about a vacation or conceptualize its merits differently when it is paired with its competing destination (Dougherty et al., 2003). Such an explanation argues for an expansion of the decision space, or the amount of information that is incorporated into the decision. This alternative hypothesis seems particularly likely within the present study, in which the choice stimuli are richly detailed and prone to trigger many associations.

Whereas this memory-driven account of the data involves stimulus elaboration and expansion of the decision space, it also seems feasible that contextual updating during the decision could result in simplification of decision space. Consistent with this notion, a large set of behavioral studies shows consistent preference reversals between decisions made in a single evaluation or a joint evaluation mode (Hsee et al., 1999). In Sharot et al., when rating vacations in isolation, participants could focus on several cognitive aspects like feasibility of travel, things to do at each destination, travel time and others in addition to pure hedonic preferences. While the behavioral ratings would represent an integrated preference across all these factors, they could still differ in terms of the underlying neural mechanisms (McClure et al., 2004). Subsequently, when forced to evaluate between two similarly rated vacations, subjects may explicitly focus on attributes that help discriminate between the two destinations, thus limiting the decision space. This shift could result in a greater reliance on pure hedonic preferences, especially given the hypothetical nature of the task and hence, subjects tend to choose destinations that are associated with greater hedonic value relative to other cognitive aspects (i.e. a vacation in Thailand might be lot more attractive than Greece when you do not have to consider factors like long travel times and cost). This increased emphasis on hedonic aspects for subsequently selected destinations might explain greater activation in caudate during the pre-choice single evaluation phase. In contrast, increased pre-choice activation in the middle frontal gyrus for subsequently rejected destinations might represent the greater emphasis on cognitive and utilitarian appraisals for these destinations pre-choice, consistent with the postulated role of this region in facilitating cognitive aspects of decision making (McClure et al., 2004; Sanfey et al., 2003). Choosing a destination under this strategy may also emphasize the positive hedonic aspects of the selected stimulus, leading to greater subsequent ratings of perceived value for these destinations over the rejected ones, as well as enhanced activation in the caudate nucleus.

Several strategies could be employed to adjudicate between these competing accounts of the data in future studies. One strategy is to vary the complexity and comparability of the stimuli. If stimuli are simple and closely matched (e.g., rating and choosing between different colored M\&Ms), it is unlikely that new information would come to mind during the choice or that any decision factors are differentially emphasized, and hence any revaluations would stem from a reduction of cognitive dissonance. Another strategy is to use the neuroimaging data from the decision phase to characterize the functional networks engaged during choices that result in large post-choice changes in valuation compared to those that result in small changes. Recruitment of memory-related regions for trials that subsequently result in larger revaluation would lend preliminary support for additional information entering the decision space. Alternatively, recruitment of regions associated with conflict detection and resolution, such as the anterior cingulate cortex and lateral prefrontal cortex, might help support a cognitive dissonance account of the data. Neuroimaging data from the choice period may additionally speak to whether the caudate actively participates in value updating during the decision, or whether it is a passive reflection of hedonic value during the rating periods only. By combining these functional comparisons with manipulations designed to emphasize one account over another, such as varying the complexity 
of the choice stimuli, we can obtain confirmatory evidence about the underlying mechanisms and their neural correlates. Finally, the neuroimaging data from the pre-choice rating period could be further investigated with respect to the magnitude of subsequent revaluation. If larger revaluation is associated with greater disparity in pre-choice caudate activity between selected and rejected stimuli, then revaluation likely reflects amplification of pre-existing differences in implicit preference, whereas a smaller pre-choice disparity would support a traditional cognitive dissonance account and corresponding revaluation during the choice period. All of these approaches could help disambiguate the mechanisms contributing to post-choice revaluation.

This study by Sharot and colleagues provides an important first step in uncovering the neural correlates of post-choice revaluation. The demonstration of prechoice differences in caudate activation predicting subsequent decisions is remarkable and raises numerous questions about the underlying assumptions implicit in designs traditionally used to study these effects. However, as emphasized above, future research is necessary to delineate the underlying mechanisms contributing to post-choice changes in perceived value.

\section{ACKNOWLEDGEMENTS}

We thank Dr. Scott A. Huettel, Dr. James R. Bettman, John A. Clithero, David V. Smith and Vishnu P. Murty for helpful comments and discussions regarding this commentary. We would also like to thank the participants of the CCN Student Journal Club at Duke University for their comments and feedback on the original article.

\section{REFERENCES}

Balleine, B. W., Delgado, M. R., and Hikosaka, O. (2007) The role of the dorsal striatum in reward and decisionmaking. J. Neurosci. 27, 8161-8165.

Brehm,J.W. (1956). Postdecision changes in the desirability of alternatives. J. Abnorm. Soc. Psychol. 52, 384-389.

Dougherty, M. R. P., Gronlund, S. D., and Gettys, C. F. (2003). Memory as a fundamental heuristic for decision making. In Emerging Perspectives on Judgment and Decision Research, S. L. Schneider and J. Shanteau, eds, (Cambridge, Cambridge University Press), pp. 125-164.

Hare, T. A., O’Doherty, J., Camerer, C. F., Schultz, W., and Rangel, A. (2008). Dissociating the role of the orbitofrontal cortex and the striatum in the computation of goal values and prediction errors. J. Neurosci. 28, 5623-5630.
Hsee, C. K., Loewenstein, G. F., Blount, S., and Bazerman, M.H. (1999). Preference reversals between joint and separate evaluations of options: a review and theoretical analysis. Psychol. Bull. 125, 576-590.

McClure, S. M., Laibson, D. I., Loewenstein, G., and Cohen, J. D. (2004). Separate neural systems value immediate and delayed monetary rewards. Science 306, 503-507.

O’Doherty, J., Dayan, P., Schultz, J., Deichmann, R., Friston, K., and Dolan, R. J. (2004). Dissociable roles of ventral and dorsal striatum in instrumental conditioning. Science 304, 452-454.

Sanfey, A. G., Rilling, J. K., Aronson, J. A., Nystrom, L. E., and Cohen, J.D. (2003). The neural basis of economic decision-making in the ultimatum game. Science 300, 1755-1758.

Seymour, B., Daw, N., Dayan, P., Singer, T., and Dolan, R. (2007).Differential encoding of losses and gains in the human striatum. J. Neurosci. 27, 4826-4831.

Sharot, T., De Martino, B., and Dolan, R. J. (2009). How choice reveals and shapes expected hedonic outcome. J. Neurosci. 29, 3760-3765.

Yin, H. H., and Knowlton, B. J. (2006). The role of the basal ganglia in habit formation. Nat. Rev. 7, 464-476.

Received: 30 June 2009; published online: 05 August 2009. Citation: Front. Hum. Neurosci. (2009) 3:18. doi: 10.3389/neuro.09.018.2009

Copyright (C) 2009 Venkatraman, Ritchey and Reeck. This is an open-access publication subject to an exclusive license agreement between the authors and the Frontiers Research Foundation, which permits unrestricted use, distribution, and reproduction in any medium, provided the original authors and source are credited. 